Article

\title{
Nonlinear Position Stabilizing Control with Active Damping Injection Technique for Magnetic Levitation Systems
}

\author{
Seok-Kyoon Kim $\mathbb{D}$ \\ Department of Creative Convergence Engineering, Hanbat National University, Daejeon 341-58, Korea; \\ lotus45kr@gmail.com or lotus45kr@hanbat.ac.kr; Tel.: +82-042-828-8801
}

Received: 25 January 2019; Accepted: 13 February 2019; Published: 17 February 2019

\begin{abstract}
This proposal suggests a novel nonlinear position-stabilizing controller for magnetic levitation (MAGLEV) applications. The proposed scheme is devised by combining the active damping injection technique and disturbance observers (DOBs), considering the inherent nonlinear dynamics, as well as parameter and load variations. The convergence and performance recovery properties are obtained by analyzing the closed-loop dynamics, which is the main contribution. The numerical verification confirms a considerable closed-loop robustness improvement, compared with the cascade-type feedback-linearization controller.
\end{abstract}

Keywords: MAGLEV; position tracking control; active damping; disturbance observer

\section{Introduction}

Magnetic levitation (MAGLEV) systems utilize an electromagnetic force to control the system body position. Due to the beneficial properties, noise reduction, and high durability and reliability, there are number of industrial applications, such as bearing position control system, MAGLEV passenger train, and so on [1-6]. However, the position control problem for MAGLEV systems is not trivial because of the inherent open-loop instability, strong nonlinearity, and a wide-range of load variations [7-9].

The MAGLEV system dynamics are strongly nonlinear, but they can be separated into slow mechanical dynamics and fast electrical dynamics [10,11]. Using this concept and linearization technique, a simple proportional-integral (PI) regulator was used to stabilize the current-loop (inner) and velocity/position-loop (outer) with a well-tuned feedback gain obtained by the time and frequency domain analysis [12]. The widening feasible operating region issue was handled by the additional gain-scheduling algorithm [13], particle swarm optimization [14], the state-feedback technique with convex optimization [15], and the adaptation algorithm for updating feedback gains [5]. These techniques still required the use of a linearized model of MAGLEV systems. There have been novel nonlinear control strategies without model linearization techniques, such as back-stepping [16], adaptive [17], sliding mode [18], fuzzy [19], coordinate transformation [20], nonlinear damping [6], and disturbance observer (DOB) techniques [21]. The stabilization region was considerably widened by these nonlinear control algorithms since the controller effectively eliminates or dominates the nonlinearities of MAGLEV system dynamics. An additional gain scheduling algorithm is still required to ensure a consistent closed-loop performance in spite of load variations coming from passengers, which corresponds to the motivation of this study.

The proposed nonlinear algorithm is designed to control the MAGLEV position robustly in the presence of parameter and load uncertainties. The advantages of this proposal fall into three parts. First, the damping injection technique in [22] is introduced so that the closed-loop transfer-function for innerand outer-loops becomes the desired first-order low-pass filter (LPF) by pole-zero cancellations. Second, 
the DOB is designed so that it estimates the time-varying disturbances coming from model-plant mismatches and load variations, exponentially. Third, based on the Lyapunov stability theorem, the closed-loop behavior is rigorously analyzed to provide the closed-loop stability and performance recovery property, which can eliminate the use of an additional gain scheduling algorithm. The realistic numerical simulation results verify the effectiveness of the proposed algorithm by showing the closed-loop robustness improvement with MATLAB/Simulink.

\section{MAGLEV Nonlinear Behaviors}

The mechanical and electrical behaviors of the MAGLEV system depicted in Figure 1 can be described by a set of nonlinear differential equations given by [5]:

$$
\begin{aligned}
\dot{d}_{m}(t) & =v_{m}(t), \\
\dot{v}_{m}(t) & =\left(\frac{M_{m}+M_{\text {load }}}{M_{m}}\right) g-\frac{K_{m}}{M_{m}} \frac{i_{c}^{2}(t)}{d_{m}^{2}(t)}+\frac{f_{\text {ext }}(t)}{M_{m}}, \\
\dot{i}_{c}(t) & =-\frac{R_{c}}{L_{c}} i_{c}(t)+\frac{1}{L_{c}} v_{c}(t), \forall t \geq 0,
\end{aligned}
$$

where the distance of $d_{m}(t)$, velocity of $v_{m}(t)$, and coil (inductor) current of $i_{c}(t)$ are treated as the state-variables and the voltage of $v_{\mathcal{C}}(t)$ applied to the inductor is used as the control input. The signal of $f_{\text {ext }}(t)$ models the external disturbance to the steel ball. The mechanical coefficients of $M_{m}, M_{\text {load }}$, $g$, and $K_{m}$ represent the steel ball mass, load mass to the steel ball, and electromagnet force constant, respectively. The electrical coefficients of $R_{c}$ and $L_{c}$ stand for the resistance and inductance values in the inductor, respectively. Thus, the load mass of $M_{\text {load }}$ and external disturbance of $f_{\text {ext }}(t)$ act as the unknown load to be systematically treated by the control action.

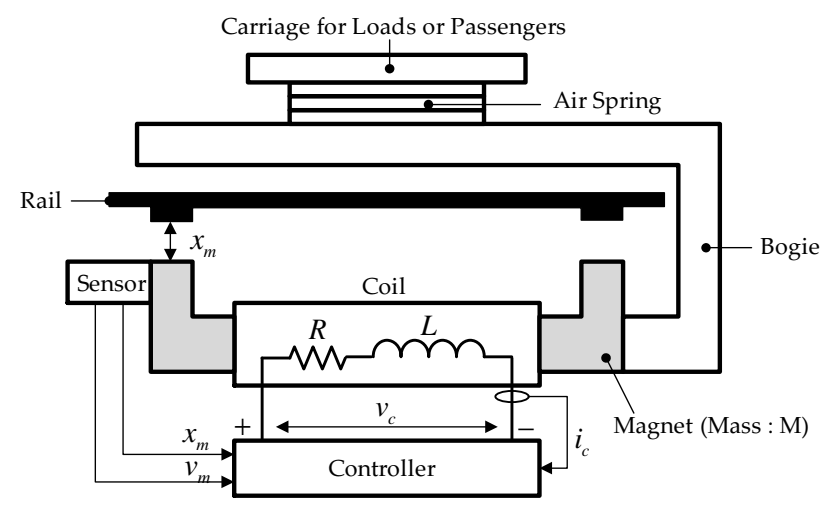

Figure 1. MAGLEV system configuration.

The consideration of coefficient and load uncertainties results in a perturbed nonlinear dynamics of (1)-(3) as:

$$
\begin{aligned}
\dot{d}_{m}(t) & =v_{m}(t) \\
\dot{v}_{m}(t) & =g-\frac{K_{m, 0}}{M_{m, 0}} \frac{i_{c}^{2}(t)}{d_{m}^{2}(t)}+w_{v, 0}(t), \\
\dot{i}_{c}(t) & =-\frac{R_{c, 0}}{L_{c, 0}} i_{c}(t)+\frac{1}{L_{c, 0}} v_{c}(t)+w_{c, 0}(t), \forall t \geq 0,
\end{aligned}
$$

with nominal coefficients of $K_{m, 0}, M_{m, 0}, R_{c, 0}$, and $L_{c, 0}$ of their true values of $K_{m}, M_{m}, R_{c}$, and $L_{s}$, respectively. The two signals of $w_{v, 0}(t)$ and $w_{c, 0}(t)$ model the external disturbances from model-plant mismatches and load variations. This study designs a position tracking controller using the perturbed nonlinear dynamics of (4)-(6) in the following section. 


\section{Position Stabilizing Controller Design}

The control objective for this section is given by:

$$
\lim _{t \rightarrow \infty} d_{m}(t)=d_{m}^{*}(t)
$$

for a given desired trajectory of $d_{m}^{*}(t)$ from the first-order low-pass filter (LPF):

$$
\dot{d}_{m}^{*}(t)=\omega_{d c}\left(d_{m, r e f}(t)-d_{m}^{*}(t)\right), \forall t \geq 0,
$$

with the desired cut-off frequency of $\omega_{d c}>0$ and the reference distance of $d_{m, r e f}(t), \forall t \geq 0$. The dynamical equation of (8) is called the target dynamics in this article, which can be used as the desired performance for tracking applications. Note that the position regulation problem can be also handled by the control objective of (7) since the combination of (7) and (8) leads to:

$$
\lim _{t \rightarrow \infty} d_{m}(t)=d_{m}(\infty)=d_{m, r e f}(\infty) .
$$

To achieve the control objective of (7), Sections 3.1 and 3.2 clearly present the outer- and inner-loop controllers for an easy implementation in a sequential manner. Section 3.3 analyzes the closed-loop behaviors in which the convergence and performance recovery properties are provided.

\subsection{Outer-Loop Design}

\subsubsection{Position Controller}

First, rewrite the position dynamics of (4) as:

$$
\dot{d}_{m}(t)=v_{m}(t)=v_{m, r e f}(t)-\tilde{v}_{m}(t), \forall t \geq 0,
$$

with $\tilde{v}_{m}(t):=v_{m, r e f}(t)-v_{m}(t)$ and the velocity reference of $v_{m, r e f}(t), \forall t \geq 0$, which can be stabilized by the simple proportional-type position controller:

$$
v_{m, r e f}(t)=\omega_{d c} \tilde{d}_{m}(t), \omega_{d c}>0, \forall t \geq 0,
$$

with $\tilde{d}_{m}(t):=d_{m, r e f}(t)-d_{m}(t), \forall t \geq 0$, and the design parameter of $\omega_{d c}>0$ assigning the cut-off frequency from the reference of $d_{m, r e f}(t)$ to the output of $d_{m}(t)$.

The substitution of (10) with (9) leads to the closed-loop position dynamics as:

$$
\dot{d}_{m}(t)=\omega_{d c} \tilde{d}_{m}(t)-\tilde{v}_{m}(t), \forall t \geq 0,
$$

which is used in Section 3.3.

\subsubsection{Velocity Controller}

The dynamics of $\tilde{v}_{m}(t)$ can be obtained by using the velocity dynamics of (5) as:

$$
\begin{aligned}
\dot{\tilde{v}}_{m}(t) & =-g+\frac{K_{m, 0}}{M_{m, 0}} \frac{i_{c}^{2}(t)}{d_{m}^{2}(t)}+w_{v}(t) \\
& =-g+\frac{K_{m, 0}}{M_{m, 0}} \frac{i_{c}^{2}(t)}{d_{m}^{2}(t)}-i_{c}(t)-\tilde{i}_{c}(t)+i_{c, r e f}(t)+w_{v}(t), \forall t \geq 0,
\end{aligned}
$$


with $w_{v}(t):=\dot{v}_{m, r e f}(t)-w_{v, 0}(t)$ and $\tilde{i}_{c}(t):=i_{c, r e f}(t)-i_{c}(t), \forall t \geq 0$. This manipulation is introduced to reduce the controller nonlinearities. A stabilizing solution to (12) is suggested with $\tilde{v}_{m, r e f}(t):=0$, $\forall t \geq 0$, as:

$$
\begin{aligned}
i_{c, \text { ref }}(t)= & g-\frac{K_{m, 0}}{M_{m, 0}} \frac{i_{c}^{2}(t)}{d_{m}^{2}(t)}+i_{c}(t)-\hat{w}_{v}(t) \\
& -k_{d} \tilde{v}_{m}(t)+\omega_{v c}\left(\tilde{v}_{m, r e f}(t)-\tilde{v}_{m}(t)\right)+k_{d} \omega_{v c} \int_{0}^{t}\left(\tilde{v}_{m, r e f}(\tau)-\tilde{v}_{m}(\tau)\right) d \tau, \omega_{v c}>0,
\end{aligned}
$$

$\forall t \geq 0$, with the DOB:

$$
\begin{aligned}
& \dot{z}_{v}(t)=-l_{v} z_{v}(t)-l_{v}^{2} \tilde{v}_{m}(t)+l_{v}\left(g-\frac{K_{m, 0}}{M_{m, 0}} \frac{i_{c}^{2}(t)}{d_{m}^{2}(t)}\right), \\
& \hat{w}_{v}(t)=z_{v}(t)+l_{v} \tilde{v}_{m}(t), l_{v}>0, \forall t \geq 0,
\end{aligned}
$$

where the design parameter of $\omega_{v c}>0$ assigns the cut-off frequency from the reference of $\tilde{v}_{m, r e f}(t)=0$ to the output of $\tilde{v}_{m}(t)=0$. The active damping term of $k_{d} \tilde{v}_{m}(t)$ is injected in the control law of (13) with $k_{d}>0$ to turn the closed-loop behaviors into a first-order dynamics from the input of $\tilde{v}_{m, r e f}(t)$ to the output of $\tilde{v}_{m}(t)$ via pole-zero cancellations. For details, see Section 3.3.

\subsection{Inner-Loop Design}

The dynamics of $\tilde{i}_{c}(t)$ can be obtained by using the current dynamics of (6) as:

$$
\dot{\tilde{i}}_{\mathcal{c}}(t)=\frac{R_{c, 0}}{L_{c, 0}} i_{c}(t)-\frac{1}{L_{c, 0}} v_{c}(t)+w_{c}(t), \forall t \geq 0,
$$

with $w_{\mathcal{c}}(t):=\dot{i}_{c, \text { ref }}(t)-w_{c, 0}(t), \forall t \geq 0$. A stabilizing solution to (16) is suggested with $\tilde{i}_{c, r e f}(t):=0$, $\forall t \geq 0$, as:

$$
\begin{aligned}
v_{c}(t)= & L_{c, 0}\left(\frac{R_{c, 0}}{L_{c, 0}} i_{c}(t)+k_{d c} \tilde{i}_{c}(t)-\omega_{c c}\left(\tilde{i}_{c, r e f}(t)-\tilde{i}_{c}(t)\right)\right. \\
& \left.-k_{d c} \omega_{c c} \int_{0}^{t}\left(\tilde{i}_{c, r e f}(\tau)-\tilde{i}_{c}(\tau)\right) d \tau+\hat{w}_{c}(t)\right), \omega_{c c}>0, \forall t \geq 0,
\end{aligned}
$$

with the DOB:

$$
\begin{aligned}
& \dot{z}_{c}(t)=-l_{c} z_{c}(t)-l_{c}^{2} \tilde{i}_{c}(t)+l_{c}\left(-\frac{R_{c, 0}}{L_{c, 0}} i_{c}(t)+\frac{1}{L_{c, 0}} v_{c}(t)\right), \\
& \hat{w}_{c}(t)=z_{c}(t)+l_{c} \tilde{i}_{c}(t), l_{c}>0, \forall t \geq 0
\end{aligned}
$$

where the design parameter of $\omega_{c c}>0$ assigns the cut-off frequency from the reference of $\tilde{i}_{c, \text { ref }}(t)=0$ to the output of $\tilde{i}_{c}(t)=0$. The active damping term of $k_{d c} \tilde{i}_{c}(t)$ is injected in the control law of (17) with $k_{d c}>0$ to turn the closed-loop behaviors into a first-order dynamics from the input of $\tilde{i}_{c, \text { ref }}(t)$ to the output of $\tilde{i}_{c}(t)$ via pole-zero cancellations. For details, see Section 3.3. Figure 2 depicts the control system structure. 


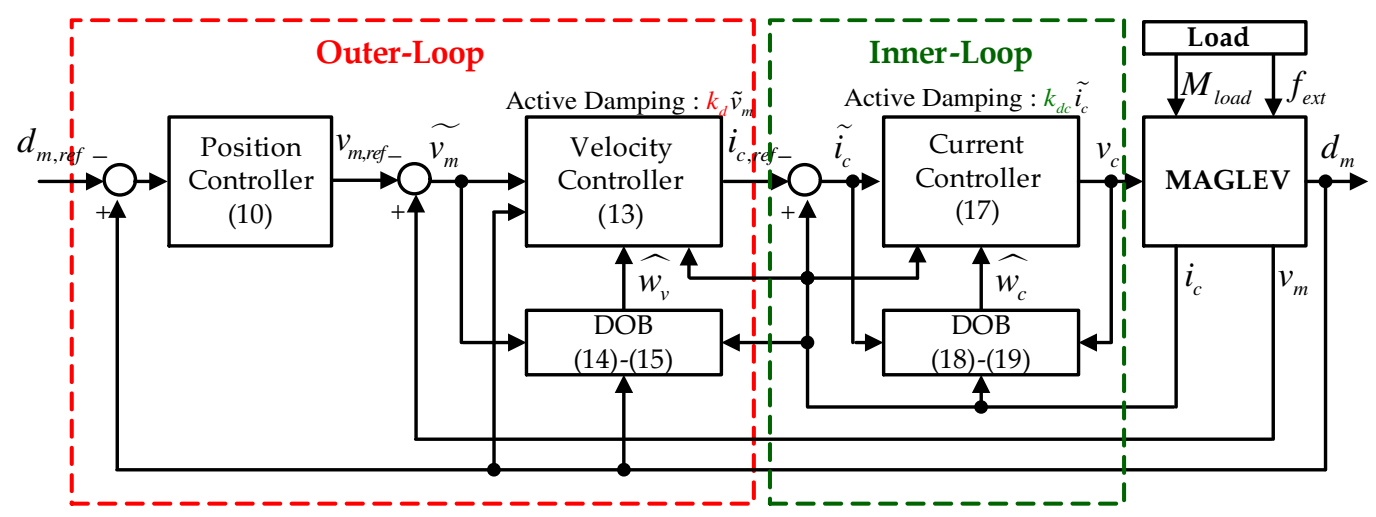

Figure 2. Control system structure.

\subsection{Analysis}

Before analyzing the closed-loop properties, it is necessary to derive the closed-loop velocity and current error dynamics, which are provided in Lemmas 1 and 2.

Lemma 1. The proposed current reference of (13) renders the velocity error dynamics of (12) to be governed by:

$$
\dot{\tilde{v}}_{m}(t)=\omega_{v c}\left(\tilde{v}_{m, r e f}(t)-\tilde{v}_{m}(t)\right)-\tilde{i}_{c}(t)+\tilde{w}_{v}(t), \forall t \geq 0,
$$

with $\tilde{v}_{m, r e f}(t)=0, \forall t \geq 0$.

Proof. Consider the closed-loop dynamics obtained by substitution of (13) with (12) as:

$$
\begin{aligned}
\ddot{\tilde{v}}_{m}(t)= & -\left(k_{d}+\omega_{v c}\right) \dot{\tilde{v}}_{m}(t)-k_{d} \omega_{v c} \tilde{v}_{m}(t)+\omega_{v c} \dot{\tilde{v}}_{m, r e f}(t)+k_{d} \omega_{v c} \tilde{v}_{m, r e f}(t) \\
& -\dot{\tilde{i}}_{c}(t)+\dot{\tilde{w}}_{v}(t), \forall t \geq 0,
\end{aligned}
$$

whose Laplace transform, $f(t) \mapsto F(s)=\mathcal{L}\{f(t)\}$, is given by:

$$
\left(s^{2}+\left(k_{d}+\omega_{v c}\right) s+k_{d} \omega_{v c}\right) \tilde{V}_{m}(s)=\omega_{v c}\left(s+k_{d}\right) \tilde{V}_{m, r e f}(s)+s\left(-\tilde{I}_{c}(s)+\tilde{W}_{v}(s)\right), \forall s \in \mathbb{C} .
$$

The factorization of $\left(s^{2}+\left(k_{d}+\omega_{v c}\right) s+k_{d} \omega_{v c}\right)=\left(s+k_{d}\right)\left(s+\omega_{v c}\right)$ renders (21) to be:

$$
\begin{aligned}
\left(s+\omega_{v c}\right) \tilde{V}_{m}(s) & =\omega_{v c} \tilde{V}_{m, r e f}(s)+\frac{s}{s+k_{d}}\left(-\tilde{I}_{c}(s)+\tilde{W}_{v}(s)\right) \\
& =\omega_{v c} \tilde{V}_{m, r e f}(s)+\left(1-\frac{k_{d}}{s+k_{d}}\right)\left(-\tilde{I}_{c}(s)+\tilde{W}_{v}(s)\right), \forall s \in \mathbb{C},
\end{aligned}
$$

via pole-zero cancellations, which gives the closed-loop velocity dynamics by taking the inverse Laplace transform as:

$$
\begin{aligned}
\dot{\tilde{v}}_{m}(t) & =\omega_{v c}\left(\tilde{v}_{m, r e f}(t)-\tilde{v}_{m}(t)\right)-\tilde{i}_{c}(t)+\tilde{w}_{v}(t)-k_{d} e^{-k_{d} t}\left(-\tilde{i}_{c}(t)+\tilde{w}_{v}(t)\right) \\
& \approx \omega_{v c}\left(\tilde{v}_{m, r e f}(t)-\tilde{v}_{m}(t)\right)-\tilde{i}_{c}(t)+\tilde{w}_{v}(t), \forall t \geq 0,
\end{aligned}
$$

where the exponential convergence property of $k_{d} e^{-k_{d} t}\left(-\tilde{i}_{c}(t)+\tilde{w}_{v}(t)\right) \rightarrow 0$ justifies the approximation above. 
The Laplace transform of resulting equation of (20) can be obtained with the assumption of $\tilde{i}_{c}(t)=\tilde{\omega}_{v}(t)=0, \forall t \geq 0$, as:

$$
\frac{\tilde{V}_{m}(s)}{\tilde{V}_{m, r e f}(s)}=\frac{\omega_{v c}}{s+\omega_{v c}}, \forall s \in \mathbb{C}
$$

where $\mathcal{L}\left\{\tilde{v}_{m, r e f}(t)\right\}=\tilde{V}_{m, r e f}(s)$ and $\mathcal{L}\left\{\tilde{v}_{m}(t)\right\}=\tilde{V}_{m}(s)$, which shows that the design parameter of $\omega_{v c}$ can be tuned for the first-order LPF of (22) with the desired cut-off frequency in rad/s.

Lemma 2. The proposed control input of (17) renders the current error dynamics of (16) to be governed by:

$$
\dot{\tilde{i}}_{c}(t)=\omega_{c c}\left(\tilde{i}_{c, r e f}(t)-\tilde{i}_{c}(t)\right)+\tilde{w}_{c}(t), \forall t \geq 0,
$$

with $\tilde{i}_{c, r e f}(t)=0, \forall t \geq 0$.

Proof. Consider the closed-loop dynamics obtained by substitution of (17) with (16) as:

$$
\ddot{\tilde{i}}_{c}(t)=-\left(k_{d c}+\omega_{c c}\right) \dot{\tilde{i}}_{c}(t)-k_{d c} \omega_{c c} \tilde{i}_{c}(t)+\omega_{c c} \dot{\tilde{c}}_{c, r e f}(t)+k_{d c} \omega_{c c} \tilde{i}_{c, r e f}(t)+\dot{\tilde{w}}_{c}(t), \forall t \geq 0,
$$

whose Laplace transform is given by:

$$
\left(s^{2}+\left(k_{d c}+\omega_{c c}\right) s+k_{d c} \omega_{c c}\right) \tilde{I}_{c}(s)=\omega_{c c}\left(s+k_{d c}\right) \tilde{I}_{s, r e f}(s)+s \tilde{W}_{c}(s), \forall s \in \mathbb{C} .
$$

The factorization of $\left(s^{2}+\left(k_{d c}+\omega_{c c}\right) s+k_{d c} \omega_{c c}\right)=\left(s+k_{d c}\right)\left(s+\omega_{c c}\right)$ renders (24) to be:

$$
\begin{aligned}
\left(s+\omega_{c c}\right) \tilde{I}_{c}(s) & =\omega_{c c} \tilde{I}_{s, r e f}(s)+\frac{s}{s+k_{d c}} \tilde{W}_{c}(s) \\
& =\omega_{c c} \tilde{I}_{s, r e f}(s)+\left(1-\frac{k_{d c}}{s+k_{d c}}\right) \tilde{W}_{c}(s), \forall s \in \mathbb{C},
\end{aligned}
$$

via pole-zero cancellations, which gives the closed-loop velocity dynamics by taking the inverse Laplace transform as:

$$
\begin{aligned}
\dot{\tilde{i}}_{c}(t) & =\omega_{c c}\left(\tilde{i}_{c, r e f}(t)-\tilde{i}_{c}(t)\right)+\tilde{w}_{c}(t)-k_{d c} e^{-k_{d c} t} \tilde{w}_{c}(t) \\
& \approx \omega_{c c}\left(\tilde{i}_{c, r e f}(t)-\tilde{i}_{c}(t)\right)+\tilde{w}_{c}(t), \forall t \geq 0,
\end{aligned}
$$

where the exponential convergence property of $k_{d c} e^{-k_{d c} t} \tilde{w}_{\mathcal{c}}(t) \rightarrow 0$ justifies the approximation above.

The Laplace transform of resulting equation of (23) can be obtained with the assumption of $\tilde{w}_{\mathcal{c}}(t)=0, \forall t \geq 0$, as:

$$
\frac{\tilde{I}_{c}(s)}{\tilde{I}_{c, r e f}(s)}=\frac{\omega_{c c}}{s+\omega_{c c}}, \forall s \in \mathbb{C}
$$

where $\mathcal{L}\left\{\tilde{i}_{c, \text { ref }}(t)\right\}=\tilde{I}_{c, \text { ref }}(s)$ and $\mathcal{L}\left\{\tilde{i}_{c}(t)\right\}=\tilde{I}_{c}(s)$, which shows that the design parameter of $\omega_{c c}$ can be tuned for the first-order LPF of (25) with the desired cut-off frequency in rad/s.

Theorem 1 asserts the closed-loop convergence property using the results of Lemmas 1 and 2 through the Lyapunov stability criterion.

Theorem 1. The closed-loop system including the proposed control law of (10), (13), (17) with DOBs of (14), (15), (18), and (19) ensures the exponential convergence:

$$
\lim _{t \rightarrow \infty} d_{m}(t)=d_{m, r e f}(t)
$$


as $\dot{d}_{m, r e f}(t) \rightarrow 0$ and $\dot{w}_{x}(t) \rightarrow 0, x=v, c$, exponentially.

Proof. The combinations of the DOB output equations of (15) and (19) and the DOB dynamics of (14) and (18) lead to:

$$
\begin{aligned}
& \dot{\hat{w}}_{v}-l_{v} \dot{\tilde{v}}_{m}=-l_{v}\left(\hat{w}_{v}-l_{v} \tilde{v}_{m}\right)-l_{v}^{2} \tilde{v}_{m}+l_{v}\left(g-\frac{K_{m, 0}}{M_{m, 0}} \frac{i_{c}^{2}}{d_{m}^{2}}\right), \\
& \dot{\hat{w}}_{c}-l_{c} \dot{\tilde{i}}_{c}=-l_{c}\left(\hat{w}_{c}-l_{c} \tilde{i}_{c}\right)-l_{c}^{2} \tilde{i}_{c}+l_{c}\left(-\frac{R_{c, 0}}{L_{c, 0}} i_{c}+\frac{1}{L_{c, 0}} v_{c}\right), \forall t \geq 0,
\end{aligned}
$$

which indicates that:

$$
\begin{aligned}
& \dot{\hat{w}}_{v}=l_{v}\left(\dot{\tilde{v}}_{m}+g-\frac{K_{m, 0}}{M_{m, 0}} \frac{i_{c}^{2}}{d_{m}^{2}}-\hat{w}_{v}\right)=l_{v}\left(w_{v}-\hat{w}_{v}\right)=l_{v} \tilde{w}_{v}, \\
& \dot{\hat{w}}_{c}=l_{c}\left(\dot{\tilde{i}}_{c}-\frac{R_{c, 0}}{L_{c, 0}} i_{c}+\frac{1}{L_{c, 0}} v_{c}-\hat{w}_{c}\right)=l_{c}\left(w_{c}-\hat{w}_{c}\right)=l_{c} \tilde{w}_{c}, \forall t \geq 0,
\end{aligned}
$$

where the two relationships of (12) and (16) are used and $\tilde{w}_{x}:=w_{x}-\hat{w}_{x}, x=v, c$. Consider the positive-definite function defined as:

$$
V:=\frac{1}{2} \tilde{d}_{m}^{2}+\frac{\kappa_{v}}{2} \tilde{v}_{m}^{2}+\frac{\kappa_{c}}{2} \tilde{i}_{c}^{2}+\sum_{x=v, c} \frac{\gamma_{x}}{2} \tilde{w}_{x}^{2}, \kappa_{x}>0, \gamma_{x}>0, x=v, c,
$$

whose time derivative can be obtained using the closed-loop trajectories of (11), (20), (23), (27), and (28) as:

$$
\begin{aligned}
\dot{V}= & \tilde{d}_{m}\left(-\omega_{d c} \tilde{d}_{m}+\tilde{v}_{m}+\dot{d}_{m, r e f}\right)+\kappa_{v} \tilde{v}_{m}\left(-\omega_{v c} \tilde{v}_{m}-\tilde{i}_{c}+\tilde{w}_{v}\right)+\kappa_{c} \tilde{i}_{c}\left(-\omega_{c c} \tilde{i}_{c}+\tilde{w}_{c}\right) \\
& +\sum_{x=v, c} \gamma_{x} \tilde{w}_{x}\left(-l_{x} \tilde{w}_{x}+\dot{w}_{x}\right) \\
\leq & -\frac{\omega_{d c}}{2} \tilde{d}_{m}^{2}-\left(\kappa_{v} \omega_{v c}-\frac{1}{2 \omega_{d c}}-1\right) \tilde{v}_{m}^{2}-\left(\kappa_{c} \omega_{c c}-\frac{\kappa_{v}^{2}}{2}-\frac{1}{2}\right) \tilde{i}_{c}^{2} \\
& -\sum_{x=v, c}\left(\gamma_{x} l_{x}-\frac{\kappa_{x}^{2}}{2}\right) \tilde{w}_{x}^{2}+\sum_{x=v, c} \gamma_{x} \dot{w}_{x} \tilde{w}_{x}+\dot{d}_{m, r e f} \tilde{d}_{m}, \forall t \geq 0,
\end{aligned}
$$

where Young's inequality, $x y \leq \frac{\epsilon}{2} x^{2}+\frac{1}{2 \epsilon} y^{2}, \forall \epsilon>0$, is applied. Now, defining $\kappa_{v}:=\frac{1}{\omega_{v c}}\left(\frac{1}{2 \omega_{d c}}+\frac{3}{2}\right)$, $\kappa_{c}:=\frac{1}{\omega_{c c}}\left(\frac{\kappa_{v}^{2}}{2}+1\right)$, and $\gamma_{x}:=\frac{1}{l_{x}}\left(\frac{\kappa_{x}^{2}}{2}+\frac{1}{2}\right), x=v, c$, it holds that:

$$
\begin{aligned}
\dot{V} & \leq-\frac{\omega_{d c}}{2} \tilde{d}_{m}^{2}-\frac{1}{2} \tilde{v}_{m}^{2}-\frac{1}{2} \tilde{i}_{c}^{2}-\sum_{x=v, c} \frac{1}{2} \tilde{w}_{x}^{2}+\sum_{x=v, c} \gamma_{x} \dot{w}_{x} \tilde{w}_{x}+\dot{d}_{m, r e f} \tilde{d}_{m} \\
& \leq-\alpha V+\sum_{x=v, c} \gamma_{x} \dot{w}_{x} \tilde{w}_{x}+\dot{d}_{m, r e f} \tilde{d}_{m}, \forall t \geq 0,
\end{aligned}
$$

with $\alpha:=\min \left\{\omega_{d c}, \frac{1}{\kappa_{v}}, \frac{1}{k_{c}}, \frac{1}{\gamma_{v}}, \frac{1}{\gamma_{c}}\right\}$. Therefore, $d_{m} \rightarrow d_{m, \text { ref }}$ as $\dot{d}_{m, \text { ref }} \rightarrow 0$ and $\dot{w}_{x} \rightarrow 0$, $x=v, c$, exponentially, since the mapping of $\left[\begin{array}{lll}\gamma_{v} \dot{w}_{v} & \gamma_{c} \dot{w}_{c} & \dot{d}_{m, r e f}\end{array}\right]^{T} \mapsto\left[\begin{array}{lll}\tilde{w}_{v} & \tilde{w}_{c} & \tilde{d}_{m}\end{array}\right]^{T}$ is strictly passive.

Finally, Theorem 2 provides the performance recovery property using the results of Lemma 1 , Lemma 2, and Theorem 1 through the Lyapunov stability criterion. 
Theorem 2. The proposed control law of (10), (13), (17) with DOBs of (14), (15), (18), and (19) forces the closed-loop system to recover exponentially the target dynamics of (8), i.e.,

$$
\lim _{t \rightarrow \infty} d_{m}(t)=d_{m}^{*}(t)
$$

as $\dot{w}_{x}(t) \rightarrow 0, x=v, c$, exponentially.

Proof. The error defined as $\tilde{d}_{m}^{*}:=d_{m}^{*}-d_{m}$ satisfies that:

$$
\dot{\tilde{d}}_{m}^{*}=-\omega_{d c} \tilde{d}_{m}^{*}+\tilde{v}_{m}, \forall t \geq 0,
$$

due to (8) and (11). Define the positive-definite function as:

$$
V^{*}:=\left.V\right|_{\tilde{d}_{m}=\tilde{d}_{m}^{*}}, \forall t \geq 0,
$$

with the function of $V$ defined in (29). Then, it follows from the closed-loop dynamics of (20), (23), (27), (28), and (32) that:

$$
\begin{aligned}
\dot{V}^{*}= & \tilde{d}_{m}^{*}\left(-\omega_{d c} \tilde{d}_{m}^{*}+\tilde{v}_{m}\right)+\kappa_{v} \tilde{v}_{m}\left(-\omega_{v c} \tilde{v}_{m}-\tilde{i}_{c}+\tilde{w}_{v}\right)+\kappa_{c} \tilde{i}_{c}\left(-\omega_{c c} \tilde{\tilde{c}}_{c}+\tilde{w}_{c}\right) \\
& +\sum_{x=v, c} \gamma_{x} \tilde{w}_{x}\left(-l_{x} \tilde{w}_{x}+\dot{w}_{x}\right) \\
\leq & -\frac{\omega_{d c}}{2}\left(\tilde{d}_{m}^{*}\right)^{2}-\left(\kappa_{v} \omega_{v c}-\frac{1}{2 \omega_{d c}}-1\right) \tilde{v}_{m}^{2}-\left(\kappa_{c} \omega_{c c}-\frac{\kappa_{v}^{2}}{2}-\frac{1}{2}\right) \tilde{i}_{c}^{2} \\
& -\sum_{x=v, c}\left(\gamma_{x} l_{x}-\frac{\kappa_{x}^{2}}{2}\right) \tilde{w}_{x}^{2}+\sum_{x=v, c} \gamma_{x} \dot{w}_{x} \tilde{w}_{x}, \forall t \geq 0,
\end{aligned}
$$

with the application of Young's inequality. The constants of $\kappa_{v}, \kappa_{c}$, and $\gamma_{x}, x=v, c$ defined in the proof of Theorem 1 yield:

$$
\begin{aligned}
\dot{V}^{*} & \leq-\frac{\omega_{d c}}{2}\left(\tilde{d}_{m}^{*}\right)^{2}-\frac{1}{2} \tilde{v}_{m}^{2}-\frac{1}{2} \tilde{i}_{c}^{2}-\sum_{x=v, c} \frac{1}{2} \tilde{w}_{x}^{2}+\sum_{x=v, c} \gamma_{x} \dot{w}_{x} \tilde{w}_{x} \\
& \leq-\alpha V^{*}+\sum_{x=v, c} \gamma_{x} \dot{w}_{x} \tilde{w}_{x}, \forall t \geq 0
\end{aligned}
$$

Therefore, $d_{m} \rightarrow d_{m}^{*}$ as $\dot{w}_{x} \rightarrow 0, x=v, c$, exponentially, since the mapping of $\left[\begin{array}{ll}\gamma_{v} \dot{w}_{v} & \gamma_{c} \dot{w}_{c}\end{array}\right]^{T} \rightarrow$ $\left[\begin{array}{ll}\tilde{w}_{v} & \tilde{w}_{c}\end{array}\right]^{T}$ is strictly passive.

\section{Simulations}

This section numerically demonstrates the advantages of the proposed controller by conducting the simulations using MATLAB/Simulink. The feedback-linearization (FL) controller, recently introduced in [23-25], was used for comparison. MAGLEV was emulated using the nonlinear differential equations of (1)-(3) with the ode 45 solver in the continuous-time variable-step setting. The control algorithms were implemented using the $C$ language-based S-function with the discrete time setting and control period of $0.1 \mathrm{~ms}$. The MAGLEV system parameters were selected as:

$$
\begin{aligned}
M_{m} & =725 \mathrm{~kg}, M_{\text {load }}=200 \mathrm{~kg}, g=9.8 \mathrm{~m} / \mathrm{sec}^{2}, \\
R_{c} & =4.4 \Omega, L_{c}=908 \mathrm{mH}, K_{m}=5.45 \times 10^{-3} \mathrm{~N} \cdot \mathrm{m}^{2} / \mathrm{A}^{2},
\end{aligned}
$$

which was given in the experimental study [5]. The nominal parameter values for controllers were set to $M_{m, 0}=0.7 M_{m}, R_{c, 0}=0.8 R_{c}, L_{c, 0}=1.3 L_{c}$, and $K_{m, 0}=1.2 K_{m}$. 
For the proposed controller, the design parameters for cut-off frequencies were adjusted as $f_{d c}=0.5, f_{v c}=10$, and $f_{c c}=30 \mathrm{~Hz}$, so that $\omega_{d c}=2 \pi f_{d c}=1.885, \omega_{v c}=62.83, \omega_{c c}=2 \pi f_{c c}=$ $188.5 \mathrm{rad} / \mathrm{s}$. The remaining design parameters were tuned as $k_{d}=k_{d c}=20$, and $l_{v}=l_{c}=628$. The feedback linearization controller, used for comparison, is given by:

$$
v_{c}(t)=L_{c, 0} \omega_{c c} \tilde{i}_{c}(t)+R_{c, 0} \omega_{c c} \int_{0}^{t} \tilde{i}_{c}(\tau) d \tau, \forall t \geq 0,
$$

with the current reference of:

$$
i_{c, r e f}(t)=\sqrt{\frac{M_{m, 0} d_{m}^{2}(t)}{K_{m, 0}}\left(g-2 \omega_{v c} \tilde{v}_{m}(t)-\omega_{v c}^{2} \int_{0}^{t} \tilde{v}_{m}(\tau) d \tau\right)}, \forall t \geq 0,
$$

where the velocity reference of $v_{m, r e f}(t)$ was set to be the same as the proposed one of (10). It is easy to see that the FL controller assigns the same cut-off frequency for inner- and outer-loops, which gives a rationale for the FL controller to be chosen for comparison.

The first stage evaluates the position tracking performance under three types of external disturbances of $f_{\text {ext }, 1}(t)=2 \times 10^{3} \sin (2 \pi 2 t), f_{\text {ext }, 2}(t)=4 \times 10^{3} \sin (2 \pi 2 t)$, and $f_{\text {ext }, 3}(t)=8 \times 10^{3} \sin (2 \pi 2 t)$. The position reference of $d_{m, r e f}(t)$ was applied in a pulse-wave form whose minimum and maximum values are $1 \mathrm{~cm}$ and $2 \mathrm{~cm}$, respectively, with the frequency of $0.1 \mathrm{~Hz}$ and the duty ratio of $50 \%$. The closed-loop position responses are presented in Figure 3, which clearly indicates that the proposed controller considerably enhances the closed-loop robustness for several types of external disturbances while maintaining tracking performance at the same level. Figures 4 and 5 show the corresponding current and DOB responses. As can be seen from Figure 4, the current over-/under-shoots are effectively reduced by the proposed controller thanks to the DOBs.

In the second stage, the position regulation performance was verified for the desired position of $1 \mathrm{~cm}$ under the external disturbance of $f_{\text {ext }, 1}=2 \times 10^{3} \sin (2 \pi 2 t)$. The load mass was increased from its initial value of $M_{\text {load }}=200 \mathrm{~kg}$ to $400 / 600 / 900 \mathrm{~kg}$, and it was restored to $M_{\text {load }}=200 \mathrm{~kg}$ in a step-wise manner. The position regulation performance comparison results are provided in Figure 6, which demonstrates that the position under-/over-shoot reduction and closed-loop robustness improvement are both accomplished by the proposed controller. Figure 7 shows the corresponding current behaviors.

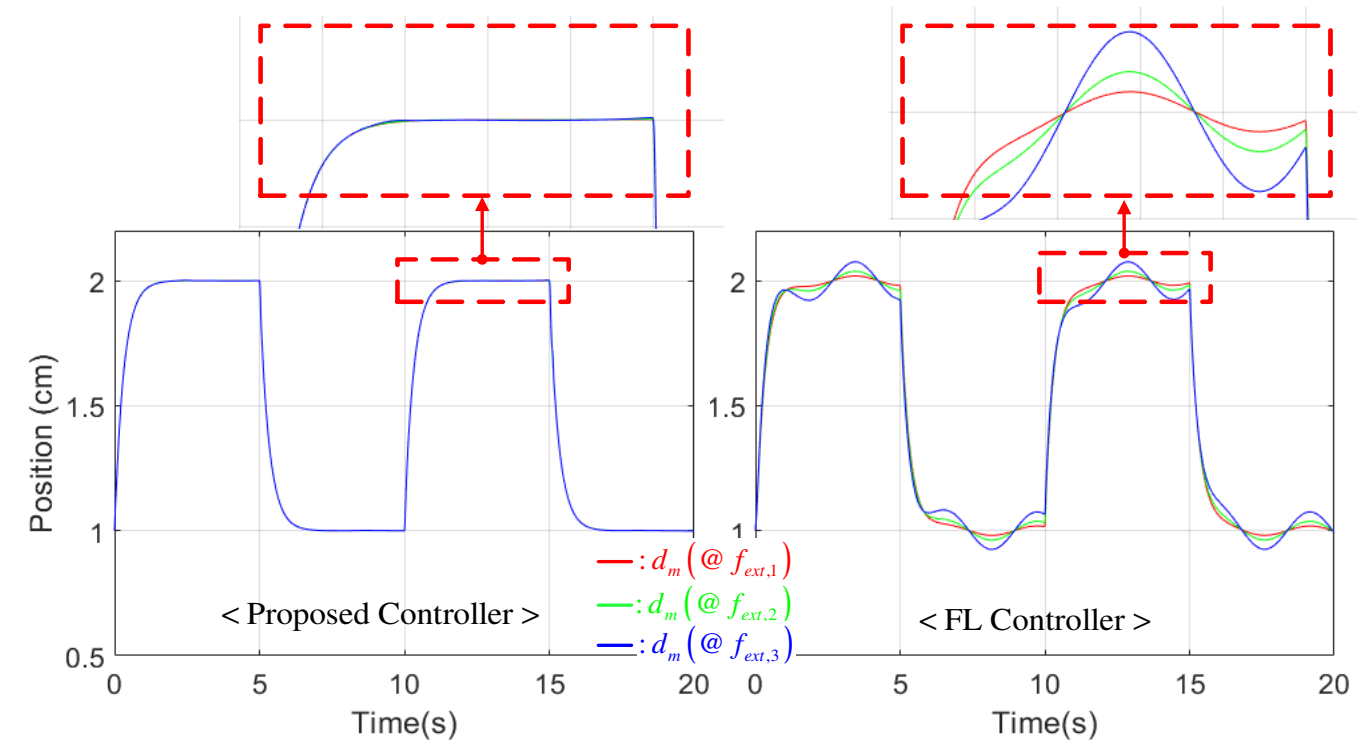

Figure 3. Position tracking performance comparison results under three types of external disturbances of $f_{\text {ext }, 1}(t), f_{\text {ext }, 2}(t)$, and $f_{\text {ext }, 3}(t)$. 

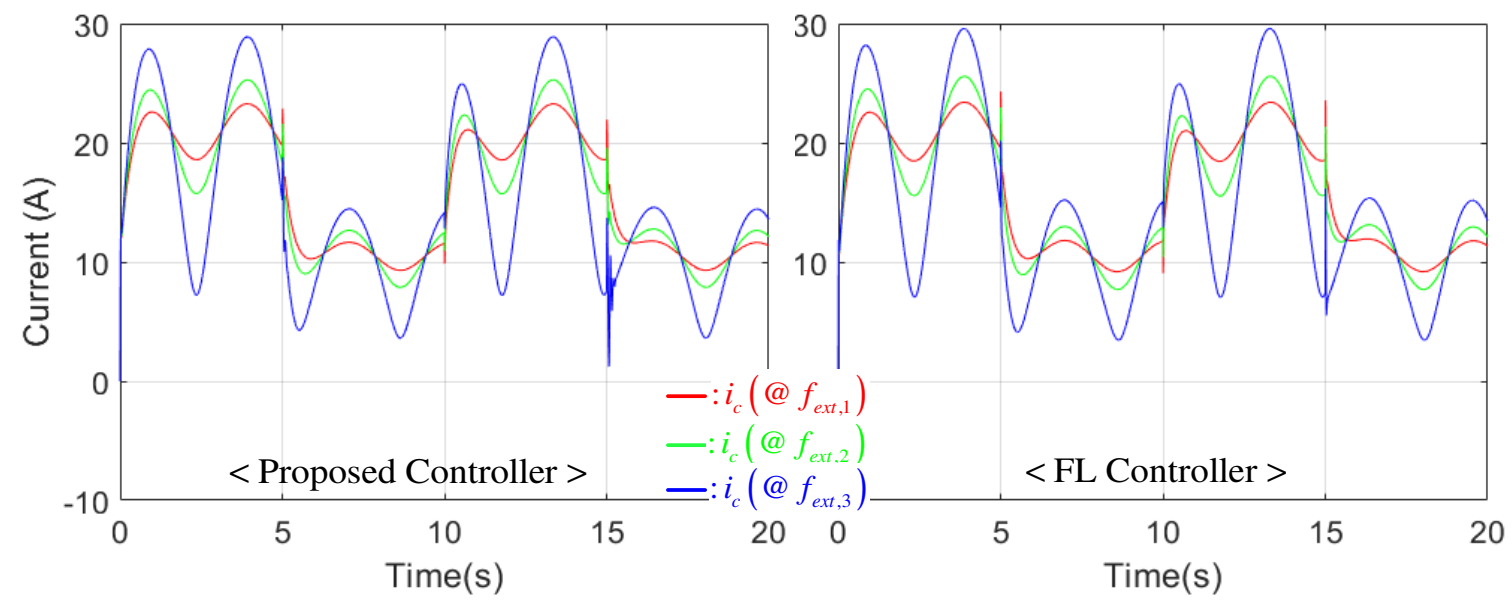

Figure 4. Current behavior comparison results under three types of external disturbances of $f_{\text {ext, } 1}(t)$, $f_{\text {ext }, 2}(t)$, and $f_{\text {ext }, 3}(t)$.
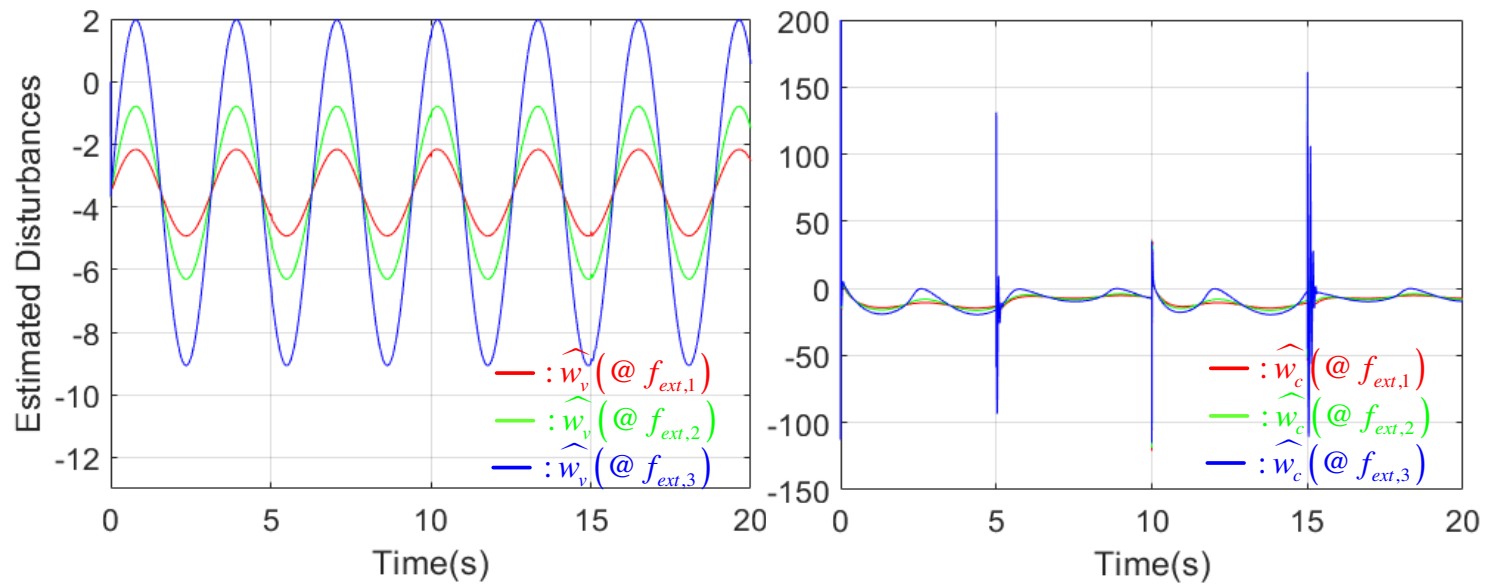

Figure 5. DOB behaviors.

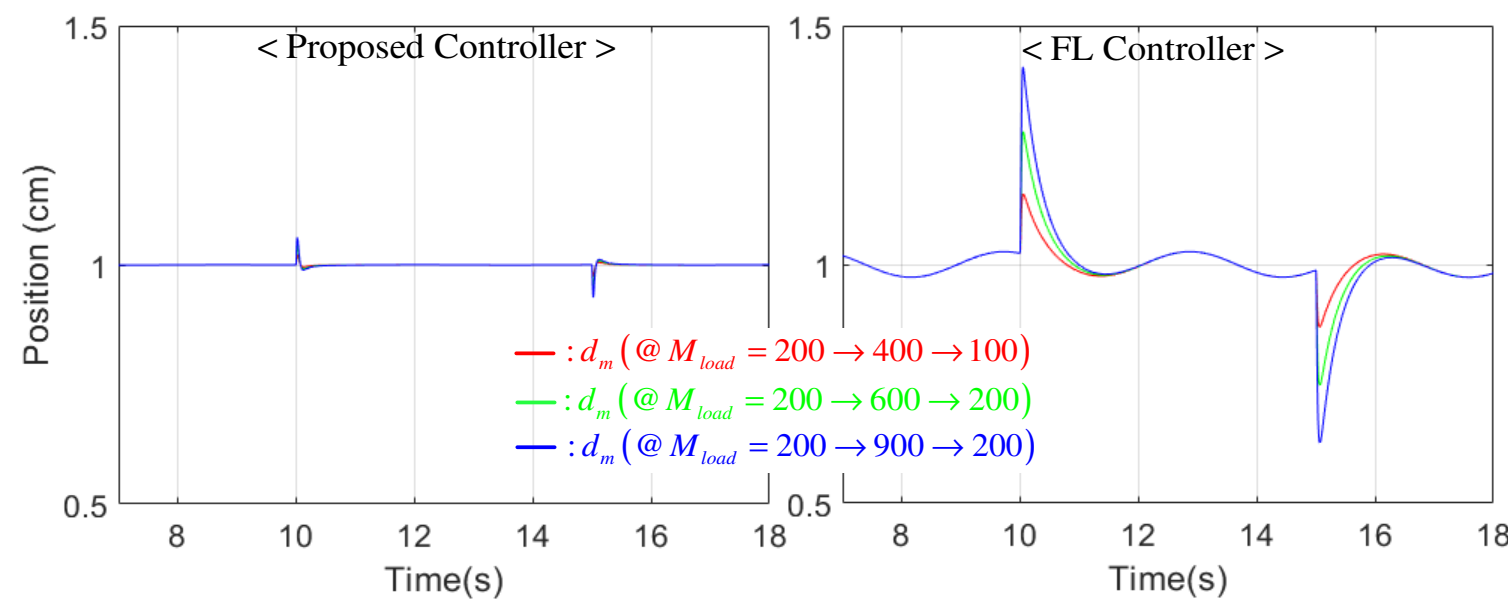

Figure 6. Position regulation performance comparison results for three load mass variation scenarios under external disturbances of $f_{\text {ext }, 1}(t)$. 

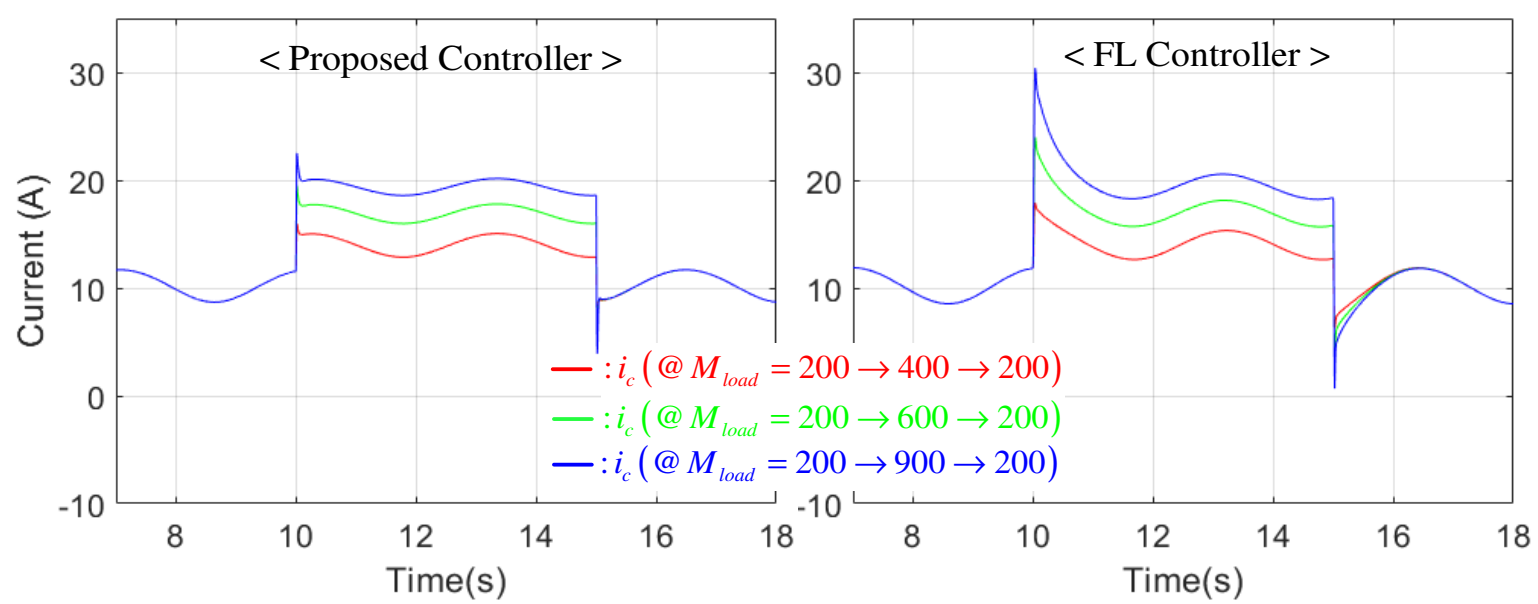

Figure 7. Current response comparison results for three load mass variation scenarios under external disturbances of $f_{\text {ext, } 1}(t)$.

The last stage investigates the closed-loop position tracking performance changes while increasing the cut-off frequency, $f_{d c}=0.1,0.2,0.5 \mathrm{~Hz}$. The external disturbance was set to $f_{\text {ext }}(t)=f_{\text {ext }, 1}(t)$. Figure 8 provides the resulting position tracking behavior change tendency. As intended, the proposed controller successfully renders the closed-loop position behaviors to be governed by the desired first-order LPF dynamics, which corresponds to the numerical evidence of the performance recovery property asserted in Theorem 2 .

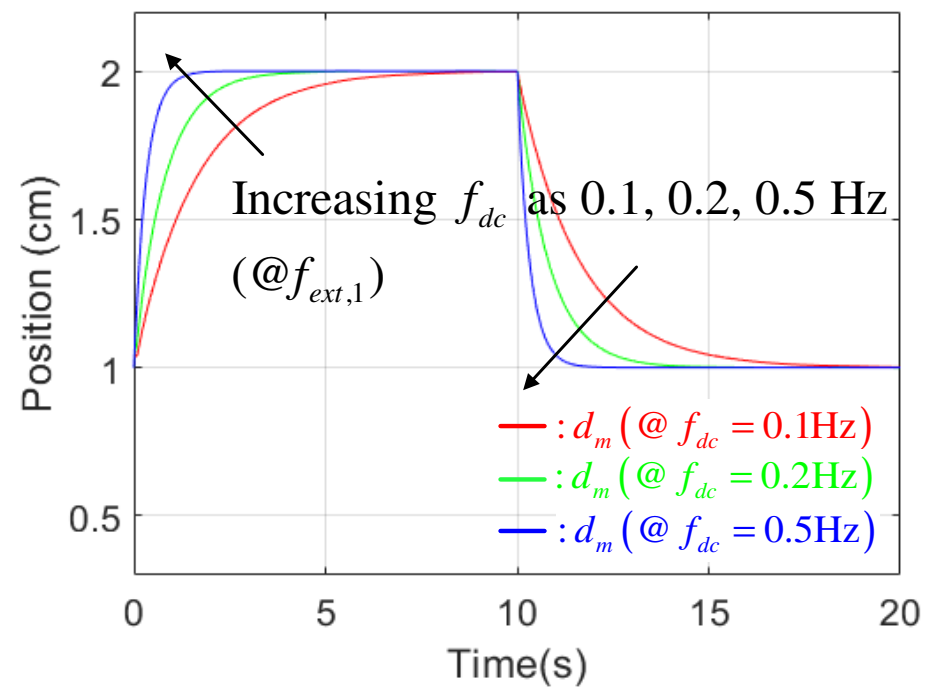

Figure 8. Position tracking performance change tendency with increasing cut-off frequency, $f_{d c}=0.1,0.2,0.5 \mathrm{~Hz}$.

\section{Conclusions}

A novel nonlinear position tracking controller was presented in this study, based on the active damping injection and DOB techniques. The parameter and load variation problems were systematically handled. The beneficial closed-loop properties were derived by rigorously analyzing the closed-loop dynamics, which was also numerically confirmed by the realistic simulations under various convincing scenarios. The simulation results also partially verified the closed-loop robustness under the model-plant mismatches from parameter and load uncertainties. A future study will investigate an optimal design parameter selection method with experimental evidence. In particular, 
the proposed method will be expanded to the general six degree-of-freedom MAGLEV systems with robustness analysis from an experimental study.

Acknowledgments: This research was supported by the research fund of Hanbat National University in 2018.

Conflicts of Interest: The author declares no conflict of interest.

\section{References}

1. Wang, Z.; Li, X.; Xie, Y.; Long, Z. MAGLEV Train Signal Processing Architecture Based on Nonlinear Discrete Tracking Differentiator. Sensors 2018, 18, 1697. [CrossRef] [PubMed]

2. Ogawa, K.; Tada, M.; Narita, T.; Kato, H. Electromagnetic Levitation Control for Bending Flexible Steel Plate: Experimental Consideration on Disturbance Cancellation Control. Actuators 2018, 7, 43. [CrossRef]

3. Wang, L.; Li, J.; Zhou, D.; Li, J. An Experimental Validated Control Strategy of MAGLEV Vehicle-Bridge Self-Excited Vibration. Actuators 2017, 7, 38. [CrossRef]

4. Wang, L.; Li, J.; Zhou, D.; Li, J. Development of Propulsion Inverter Control System for High-Speed MAGLEV based on Long Stator Linear Synchronous Motor. Energies 2017, 10, 170.

5. Zhang, Z.; Li, X. Real-Time Adaptive Control of a Magnetic Levitation System with a Large Range of Load Disturbance. Sensors 2018, 18, 1512. [CrossRef] [PubMed]

6. Yang, Z.J.; Minashima, M. Robust Nonlinear Control of a Feedback Linearizable Voltage-Controlled Magnetic Levitation System. IEEJ Trans. Electron. Inf. Syst. 2001, 121, 1203-1211. [CrossRef]

7. Zhang, L.; Campbell, S.; Huang, L. Nonlinear analysis of a MAGLEV system with time-delayed feedback control. Phys. D Nonlinear Phenom. 2012, 240, 1761-1770. [CrossRef]

8. Santos, M.; Ferreira, J.; Simoes, J.; Pascoal, R.; Torrao, J.; Xue, X.; Furlani, E. Magnetic levitation-based electromagnetic energy harvesting: A semi-analytical non-linear model for energy transduction. Sci. Rep. 2016, 6, 18579. [CrossRef] [PubMed]

9. Zhang, Z.; Zhang, L. Hopf bifurcation of time-delayed feedback control for MAGLEV system with flexible guideway. Appl. Math. Comput. 2013, 219, 6106-6112. [CrossRef]

10. Cho, H.W.; Yu, J.S.; Jang, S.M.; Kim, C.H.; Lee, J.M.; Han, H.S. Equivalent Magnetic Circuit Based Levitation Force Computation of Controlled Permanent Magnet Levitation System. IEEE Trans. Magn. 2012, 48, 4038-4041. [CrossRef]

11. Jeong, J.H.; Ha, C.W.; Lim, J.; Choi, J.Y. Analysis and Control of the Electromagnetic Coupling Effect of the Levitation and Guidance Systems for a Semi-High-Speed MAGLEV Using a Magnetic Equivalent Circuit. IEEE Trans. Magn. 2016, 52, 8300104. [CrossRef]

12. Lee, H.; Kim, K.; Lee, J. Review of MAGLEV train technologies. IEEE Trans. Magn. 2006, 42, 1917-1925.

13. Shamma, J.; Athans, M. Analysis of gain scheduled control of nonlinear plants. IEEE Trans. Autom. Control 2002, 35, 898-907. [CrossRef]

14. Wai, R.; Lee, J.; Chuang, K. Real-time PID control strategy for MAGLEV transportation system via particle swarm optimization. IEEE Trans. Ind. Electron. 2011, 58, 629-646. [CrossRef]

15. Kim, C.H. Robust Control of Magnetic Levitation Systems Considering Disturbance Force by LSM Propulsion Systems. IEEE Trans. Magn. 2017, 53, 8300805. [CrossRef]

16. Wai, R.J.; Lee, J.D. Backstepping-based levitation control design for linear magnetic levitation rail system. IET Control Theory Appl. 2008, 2, 72-86. [CrossRef]

17. Sadek, U.; Sarja, A.; Chowdhury, A.; Svečko, R. Improved adaptive fuzzy backstepping control of a magnetic levitation system based on symbiotic organism search. Appl. Soft Comput. 2017, 56, 19-33. [CrossRef]

18. Sun, Y.; Li, W.; Xu, J.; Qiang, H.; Chen, C. Nonlinear dynamic modeling and fuzzy sliding-mode controlling of electromagnetic levitation system of low-speed MAGLEV train. J. Vibroeng. 2017, 19, 328-342. [CrossRef]

19. Wai, R.J.; Lee, J.D. Robust Levitation Control for Linear MAGLEV Rail System Using Fuzzy Neural Network. IEEE Trans. Control Syst. Technol. 2009, 17, 4-14.

20. Kaloust, J.; Ham, C.; Siehling, J.; Jongekryg, E. Nonlinear robust control design for levitation and propulsion of a MAGLEV system. IET Control Theory Appl. 2004, 151, 460-464. [CrossRef]

21. Yang, J.; Zolotas, A.; Chen, W.; Michail, K.; Li, S. Robust control of nonlinear MAGLEV suspension system with mismatched uncertainties via DOBC approach. ISA Trans. 2011, 50, 389-396. [CrossRef] [PubMed] 
22. Xu, J.; Xie, S.; Tang, T. Active Damping-Based Control for Grid-Connected LCL-Filtered Inverter with Injected Grid Current Feedback Only. IEEE Trans. Ind. Electron. 2014, 61, 4746-4758. [CrossRef]

23. Zou, D.; She, L.; Zhang, Z.; Chang, W. MAGLEV system controller design based on the feedback linearization methods. In Proceedings of the 2008 International Conference on Information and Automation, Changsha, China, 20-23 June 2008.

24. Li, J.; Li, J.; Cui, P. Mass adaptation of MAGLEV levitation system based on feedback linearization. In Proceedings of the 2013 10th IEEE International Conference on Control and Automation (ICCA), Hangzhou, China, 12-14 June 2013.

25. Khalil, H.K. Nonlinear Systems; Prentice Hall: Upper Saddle River, NJ, USA, 2002.

(C) 2019 by the author. Licensee MDPI, Basel, Switzerland. This article is an open access article distributed under the terms and conditions of the Creative Commons Attribution (CC BY) license (http://creativecommons.org/licenses/by/4.0/). 